the mobilisation of hepatic TG stored, or from the reesterification of fatty acids coming from the hepatic captation of degraded lipoproteins.

Effect of butyrate on the expression of cyclin-dependent kinases and cyclin $D$ by HT-29 epithelial cells. S Siavoshian ', HM Blottière $^{2}$, C Cherbut ${ }^{2}$, B Kaeffer ${ }^{2}$, JP Galmiche ${ }^{1}\left({ }^{1}\right.$ Centre de recherche en nutrition humaine de Nantes, Hôpital Laënnec; ${ }^{2}$ Inra-LTAN, 44000 Nantes, France).

Butyrate, a four-carbon fatty acid produced by dietary fibre fermentation, can modulate colonic epithelial cell proliferation in vitro. In a variety of cell systems, butyrate has been found to block cells in $G_{1}$ phase. D-type cyclins are synthesized in early $G_{1}$, and activate cdk4 and cdk6 as cells leave the quiescent phase. Cyclin E-cdk2 and cyclin A-cdk2 complexes form later in $G_{1}$ as cells prepare to DNA synthesis. The aim of our study was to investigate the mechanisms by which butyrate inhibits cell cycle progression toward $S$ phase.

HT-29 cells were synchronized by exposing the culture to fetal calf serum-deprived DMEM medium. Cells were then exposed to complete medium ( $10 \%$ fetal calf serum) in the presence or absence of increasing concentration of sodium butyrate (from 2 to $8 \mathrm{mM}$ ) for 4 days. Proteins were extracted, and cyclin D, cdk2, cdk4 and cdk6 expression were studied by Western blotting.

After exposition to $2 \mathrm{mM}$ sodium butyrate, a strong stimulation of cyclin $\mathrm{D}$ expression was observed, on HT-29 cells and it was optimal at $8 \mathrm{mM}$. This stimulation is associated with a decreased expression of cdk2. The level of cdk4 and cdk6 remained inchanged.

The increased level of cyclin D may be a marker of the blockade in $G_{1}$ phase, because in continuously proliferating cell populations, the level of $\mathrm{D}$ type cyclins is low.
However, the decreased expression of cdk2 which leads to the inhibition of events essential for DNA replication, may participate directly to the mechanism of action of butyrate on cell proliferation.

Bacterial status is a key-factor controlling expression of the mitochondrial HMG-CoA synthase gene in the rat intestinal mucosa. C Cherbuy ${ }^{1}, \mathrm{~B}$ Darcy-Vrillon ${ }^{1}$, JP Pégorier ${ }^{2}$, C Andrieux ', B Azzout ${ }^{2}$, PH Duée ${ }^{1}{ }^{1}$ UEPSD, Inra, 78352 Jouy-en-Josas cedex; ${ }^{2}$ Ceremod, CNRS, 92190 Meudon Bellevue, France).

Butyrate from bacterial origin is a major energy substrate for colonic epithelial cells. In these cells, ketone body production from butyrate proceeds via the mitochondrial 3-hydroxy-3-methylglutaryl-CoA (HMG$\mathrm{CoA}$ ) synthase. We investigated the effect of the intestinal site, as well as the nutritional and bacterial status on this enzyme expression in the rat intestinal mucosa.

Thirty-six male rats (240-310 g) differing by their bacterial status were used at the age of 3 months: conventional (CV) rats, germ-free (GF) rats and germ-free rats inoculated (IN) at 2 months with the intestinal flora of conventional rats, by gastric administration of freshly obtained and diluted feces. All rats were allowed free access to water and diet, except for a group of $\mathrm{CV}$ rats which were fasted for $48 \mathrm{~h}$ prior to sacrifice. HMG-CoA synthase expression was quantified by northern and western blot analysis of jejunal or colonic mucosal scrapings.

The mRNA encoding HMG-CoA synthase were detected in the colonic mucosa of $\mathrm{CV}$ rats, and their concentration was only modestly decreased after a $48 \mathrm{~h}$ starvation $(-26 \pm 11 \% ; P<0.10)$. In contrast, HMG-CoA synthase was not expressed in the small intestinal mucosa, whatever the nutritional status of the animals. The concentrations of mRNA and immunoreactive 
protein were dramatically reduced in the colonic mucosa of GF vs CV rats (respectively $-55 \pm 6 \%$, and $-66 \pm 2 \%, P<0.05$ ). Moreover, these changes were reversible, as $\mathrm{mRNA}$ and protein concentrations were restored in the colonic mucosa of IN rats.

In conclusion, there is a site-specific expression of mitochondrial HMG-CoA synthase in the intestinal mucosa of the adult rat. As opposed to what happens in the liver, its expression is not greatly affected by starvation vs feeding. In contrast, the large intestinal flora, through mechanisms which deserve further investigation, is a major factor controlling the enzyme.

\section{Influence of dietary proteins on colonic cell proliferation and luminal polyamine} metabolism. K Meziani, R Benamouzig, D Tomé (Inra, UNHPI, 75006 Paris, France).

Polyamines play an important role in DNA, RNA and protein synthesis. The current study was designed to investigate the influence of the quality of dietary proteins on luminal intestinal polyamine concentrations and their possible role in colonic cell proliferation.

Three groups of eight male Wistar rats were fed high protein diets $(50 \%$ of either casein, zein or soy protein). After 4 weeks of feeding, both intestinal contents and colonic mucosa were recovered. Polyamines were assayed by HPLC and ornithine decarboxylase (ODC) activity was measured by the release of ${ }^{14} \mathrm{CO}_{2}$ from $14 \mathrm{C}$-L-ornithine.

Rats fed zein showed a 3-fold increase in mucosal colonic ODC activity compared to the other groups $(31 \pm 11,11 \pm 4$ and 10 $\pm 2 \mu \mathrm{mol} / \mathrm{h} / \mathrm{mg}$ proteins for zein, casein and soy protein groups, respectively $P<0.05$ ). Luminal colonic putrescine and cadaverine levels were also higher in the group fed zein (putrescine: $14 \pm 6,3 \pm 1$ and $0.6 \pm$ $0.3 \mu \mathrm{mol} / \mathrm{g} \mathrm{DM}$; cadaverine: $23 \pm 11,0.6 \pm$ 0.1 and $0.2 \pm 0.09 \mu \mathrm{mol} / \mathrm{g} \mathrm{DM}$ for zein, casein and soy protein groups, respectively $P<0.05$ ). Furthermore, a high amount of polyamines were found in the jejunum and the ileum of rats receiving a soy protein diet, probably due to the higher level of polyamines in this diet. The difference of the true digestibility of dietary arginine $(95 \%$ for soy protein, $93 \%$ for casein and $42 \%$ for zein), a precursor of polyamines, could explain these observations.

In conclusion, mucosal colonic ODC activity is influenced by dietary protein quality. Zein is associated with a higher colonic ODC activity. This modulation could be influenced by modifications in luminal polyamine concentrations.

\section{Uptake of $\alpha$-linolenic acid in human ente-} rocyte-like Caco- 2 cells. T Tranchant ${ }^{1}, \mathrm{P}$ Besson $^{2}$, C Hoinard ${ }^{2}$, J Delarue ${ }^{1}, \mathrm{C}$ Couet ${ }^{1}$, J Goré ${ }^{2}\left(^{1}\right.$ Laboratoire de nutrition, Faculté de médecine; ${ }^{2}$ Laboratoire de physiologie et biophysique cellulaire, Faculté de pharmacie, Université de Tours, 37000 Tours, France).

The uptake kinetics of $\alpha$-linolenic acid were investigated in the human intestinal cell line Caco-2. Four clones (PD10, PF11, PD7 and TC7) from the parental Caco-2 cells population were used. The TC7 clone was selected for the study of $\alpha$-linolenic acid uptake. $\left[1-{ }^{14} \mathrm{C}\right]$ linolenic acid dissolved in taurocholate was presented to the microvillus plasma membrane of differentiated TC7 cells. The initial rate of uptake is not a linear function of the $\alpha$-linolenic monomer concentration in the incubation medium. In the monomer concentration range studied $(0.2$ to $36 \mu \mathrm{M}$ ) apical uptake was saturable and followed Michaelis-Menten kinetics ( Vm = $15.4 \pm 0.6 \mathrm{nmol} / \mathrm{mg}$ protein $/ \mathrm{min}, \mathrm{Km}=14.3$ $\pm 1.3 \mu \mathrm{M}$ ). In addition, it was temperature and energy-dependent but was apparently unaffected by the sodium gradient. Excess of unlabeled long chain fatty acids led to a $27-68 \%$ reduction of $\left[1-{ }^{14} \mathrm{C}\right]$ linolenic acid 